\title{
Agôn
}

Revue des arts de la scène

5 | 2012

L'entrée en scène

\section{Regards croisés sur l'entrée en scène}

Entretien réalisé par Anne Pellois

Enzo Cormann, Alexandre de Dardel, Philippe Delaigue et Anne Pellois

\section{(2) OpenEdition}

Journals

Édition électronique

URL : http://journals.openedition.org/agon/2417

DOI : 10.4000/agon.2417

ISSN : 1961-8581

Éditeur

Association Agôn

Référence électronique

Enzo Cormann, Alexandre de Dardel, Philippe Delaigue et Anne Pellois, «Regards croisés sur l'entrée en scène », Agôn [En ligne], 5 | 2012, mis en ligne le 25 janvier 2013, consulté le 15 septembre 2020.

URL : http://journals.openedition.org/agon/2417

Ce document a été généré automatiquement le 15 septembre 2020.

Association Agôn et les auteurs des articles 


\title{
Regards croisés sur l'entrée en scène
}

\author{
Entretien réalisé par Anne Pellois
}

Enzo Cormann, Alexandre de Dardel, Philippe Delaigue et Anne Pellois

\section{NOTE DE L'ÉDITEUR}

Entretien réalisé le 22 juin 2012 à l'ENSATT

Enzo Cormann dirige le département d'écriture à l'ENSATT, auteur de nombreuses pièces, dont Toujours l'Orage, Cairn, La Révolte des Anges, ainsi que de deux essais, À quoi sert le théâtre? (2003) et Ce que seul le théâtre peut dire, considérations poélitiques (2012).

Alexandre de Dardel co-dirige le département scénographie de l'ENSATT. Il est scénographe au théâtre et à l'opéra et a notamment collaboré avec Jean-François Sivadier (Wozzeck en 2007, Carmen en 2010, La Traviata en 2011), Stéphane Braunschweig (Tartuffe, 2008), Laurent Gutmann (Nouvelles du Plateau S, 2003).

Philippe Delaigue dirige le département jeu de l'ENSATT. Acteur et metteur en scène (À l'Ombre en 2010, Le Grand ensemble en 2011), il est aussi fondateur de La Fédération en 2007, compagnie qui s'interroge sur l'origine du geste théâtral, qui ne serait pas l'apanage du seul metteur en scène.

\section{Apprendre à entrer}

\footnotetext{
Anne peLlols: La première question que j'aimerais poser est pour les directeurs de département que vous êtes. Est-ce que la question de l'entrée en scène se pose de manière centrale dans vos enseignements? En jeu, est-ce que la question de l'entrée de l'acteur est travaillée? En écriture, est-ce qu'on apprend à écrire une entrée en scène? Et en scénographie, est-ce que l'on travaille l'espace à partir des entrées en scène ?

Enzo CORMANN : Peut-être que celui qui n'entre jamais en scène peut commencer. La question de l'entrée en scène pour les écrivains c'est en effet la façon dont on commence.
} 


\section{AP : C'est l'incipit?}

EC: Oui. Par exemple, est-ce qu'on va commencer in medias res ou pas? Spontanément, et cela me frappe beaucoup, le paradigme qui prévaut chez les jeunes écrivains, c'est le commencement par le milieu, comme si le théâtre était une fenêtre qui s'ouvrait brutalement sur le réel, et qu'on entendait la réponse à une question. Crac! Par la magie du théâtre on a abattu un mur et on entre de plain pied dans une action.

Bien sûr, cela ne va pas sans poser problème, car on se retrouve directement dans un modèle de drame absolu - temps présent, relations interpersonnelles -, bref, dans du dramatique pur jus. C'est très compliqué de se sortir de là parce qu'on rentre dans une logique d'immédiateté, de quatrième mur, etc. Dans la mesure où il n'y a décidément plus de canons que l'on pourrait transmettre de façon pertinente et donc un dogme ou une doctrine sur lesquels on pourrait se mettre tous d'accord, on ne fait qu'interroger cette forme, souvent perçue comme une évidence. Nombre de jeunes écrivains ont tendance à se représenter l'entrée en scène comme une effraction (voire qu'il n'existe aucune alternative). Que cela va de soi, en quelque sorte. Il s'agit précisément de poser dans la discussion critique que cela ne va pas de soi, que d'ailleurs le théâtre ne va pas de soi, qu'il faut qu'il puise son énergie quelque part, qu'il puise sa légitimé d'opération spécifique dans sa façon spécifique d'aborder le réel. Cela ne va pas de soi, mais de l'autre - de l'assemblée. Et ça suppose une conscience aiguë du type d'opération qu'on embraye en commençant.

Alexandre DE DARDEL : Pour la scénographie, on travaille sans acteur, comme Enzo. Ou plutôt nos acteurs sont des figurines de quelques centimètres dans les maquettes. Pour nous aussi, c'est la question du « comment ça commence » qui est primordiale. C'est une question que je pose à mes étudiants. Mais je ferais même remonter le début au moment où le spectateur n'est pas encore dans la salle. Le spectateur entre dans le théâtre, s'assoit, et puis ça commence. Est-ce qu'il y a un rideau, pas de rideau? C'est vrai que je pose de plus en plus cette question. Après on ne se pose pas tellement la question du point de vue du concret de l'acteur, ou alors d'une manière très générale, par la question des entrées et des sorties dans l'espace. Mais nous ne travaillons pas sur la précision de l'entrée.

\section{AP : L'espace du seuil n'est pas une question qui se pose ?}

$\mathrm{AdD}$ : Non, pour moi, l'entrée en scène est quand même très liée à la mise en scène, c'est-à-dire au projet total, dont la scénographie fait partie. Mais la thématique de l'entrée en scène dans l'espace pour moi c'est très flou, sauf quand il s'agit de penser le début.

Philippe DELAIGUE: Je ne sais pas si l'entrée en scène peut faire l'objet d'un enseignement. La seule chose académique que j'ai apprise sur l'entrée, c'est Planchon qui me l'a apprise : quand quelqu'un rentre en scène, il faut que tout le monde le regarde entrer. Lui le faisait systématiquement. C'est une chose très académique. C'était comme cela pour lui qu'on pouvait voir quelqu'un entrer. Sinon on ne le voyait pas.

Dans le département, on n'enseigne pas spécifiquement l'entrée en scène. Si je dois évoquer cette chose là avec de jeunes acteurs, c'est plus dans la signification de l'entrée en scène : qu'est-ce que cela représente comme ambition de rentrer sur une scène, est-ce qu'on est à la hauteur de cette tâche-là ? Qu'est-ce que cette ambition de 
s'adresser à une communauté de gens, à un public? Que représente cet espace que l'on pénètre, quelle part de sacré, quelle valeur, quel type de territoire? Ces questions-là sont souvent évoquées et ont toutes quelque chose à voir avec la prise de pouvoir sur cet espace. Ce que j'essaye de leur dire, c'est que cet espace doit être pris. Ce n'est pas un espace dans lequel on entre par effraction, avec inhibition. Certes, la question du comment se pose, mais la question du pourquoi est encore plus importante. Pourquoi je rentre là et qu'est-ce que je vais y faire et pour parler à qui ?

Le déclenchement tonique de l'entrée, à savoir ce qui se passe dans le corps au moment où l'on va entrer et comment cela se manifeste, est également très important pour nous. Au concours d'entrée par exemple, très peu se posent cette question-là. Certains sont capables de démarrer une scène sans qu'il y ait de début. Ils sont là, puis tout à coup ça commence, mais on ne sait pas exactement quand ça a commencé dans le corps. Il s'agit d'interroger les appuis toniques, la mobilisation énergétique du corps au moment de commencer.

$A P$ : C'est une entrée en jeu?

Ph.D : Absolument. Une entrée en jeu tonique. J'insiste beaucoup sur la tonicité car on est quand même dans une époque assez molle.

L'entrée a aussi une importance en tant que seuil, puisqu'on se rend compte que le seuil est l'endroit critique des pièces et des scènes. C'est souvent sur le seuil que se passent les catastrophes. C'est souvent au moment de partir qu'on dit les choses les plus rédhibitoires. Les scènes commencent toujours au moment où il $\mathrm{y}$ en a un qui part, et qui dit la chose qui va déclencher une catastrophe et prolonger la scène. Donc le seuil c'est l'espace critique. Et l'entrée de ce point de vue-là, c'est aussi un endroit de sortie. Cet espace là pour nous il est très important. On est très souvent en train de faire des scènes dans l'entre-deux. On déplace la coulisse sur scène d'une certaine manière, et on ne cesse pas de travailler sur des seuils, puisqu'on est tout le temps en train de dire pourquoi on reste là, en fait.

AdD : Pour rebondir sur ce que disait Philippe, évidemment que c'est très important, la manière d'entrer et de sortir de scène. Mais il m'est difficile de théoriser cette question, car cela dépend tellement de la nature du spectacle et de la mise en scène. Je vais donc prendre exemple sur des spectacles sur lesquels j'ai travaillé. JeanFrançois Sivadier a monté La Traviata au festival d'Aix en Provence en 2011. Pendant le prologue, et c'est la liberté de l'opéra que de pouvoir inventer des choses pendant le prologue, il a fait rentrer sur scène Nathalie Dessay - qui jouait Violetta - d'une manière particulière. Il l'a faite rentrer par une simple porte dans un mur, même pas au centre, mais à jardin. 
La Traviata, Mise en scène de Jean-François Sivadier, scénographie Alexandre de Dardel (2011)

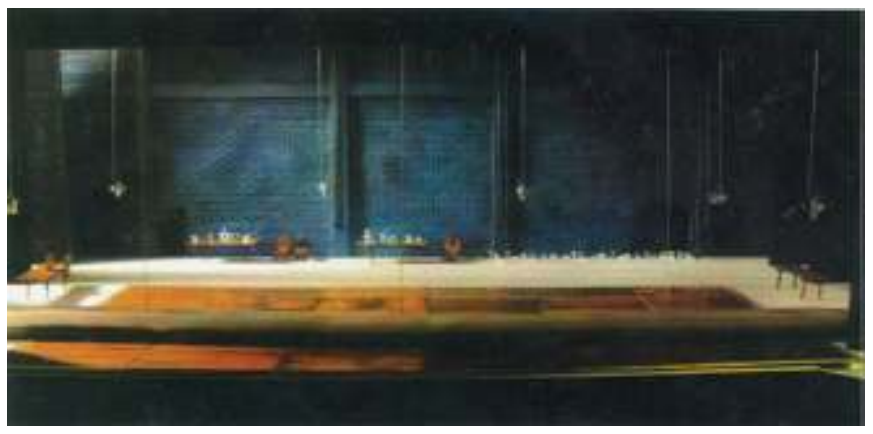

Alexandre de Dardel

Il l'a faite rentrer comme un petit clown, et on voyait aussi Giulietta Masina. On voyait donc l'héroïne de La Traviata, et en même temps on voyait Nathalie Dessay, l'actrice, qui rentre dans son théâtre. Il n'a pu le faire de cette manière que parce que c'était Nathalie Dessay. Parce que dès qu'elle passe la porte, tout le monde reconnaît Nathalie Dessay. Cette entrée en scène marche parce que l'actrice est très connue. Donc voilà, ce n'était rien du tout, juste une porte. Mais cela marchait d'autant plus qu'elle entre pour ne plus sortir de scène, pour y mourir.

D'un côté il y a ce presque rien, cette simplicité, le caractère unique de cette entrée dans le spectacle, et de l'autre côté, il y a des spectacles saturés d'entrées et de sorties. En 2003, j'ai travaillé sur Nouvelles du Plateau S, de Oriza Hirata. Avec le metteur en scène Laurent Gutmann, on s'est amusé à compter les entrées et les sorties. Il y en avait cent quatre-vingt cinq ! Ça n'arrêtait pas. Et là c'est tout autre chose, l'espace devient comme une clairière entourée d'une forêt d'où l'on sort et où l'on disparait.

\section{Registres de l'entrée}

AP: Quel est le registre de l'entrée en scène? Une apparition? Un franchissement? Un saut ? Ou bien est-ce quelque chose qui peut passer inaperçu, ne pas se voir?

EC : C'est toujours pareil. Quand on parle d'écriture, on n'est pas dans la matérialité immédiate. La question - et cela fait écho à ce que disait Philippe à l'instant -, c'est où débarque-t-on, et d'où vient-on? Il faut avoir cette conscience-là. L'écriture ne peut pas faire comme si elle n'avait pas à se soucier de le savoir. Même si de façon intuitive on peut arriver à des propositions véritablement inouïes, poétiques, bouleversantes. Mais dès que la discussion s'organise, dès que le projet se conscientise, dès qu'on porte un regard critique là-dessus, on ne peut pas manquer de voir ce que c'est que cette effraction, consentie certes, mais enfin effraction tout de même, suscitée par le désir de conduire une expérience pour le compte d'une assemblée qui n'a pas passé une commande précise, mais qui est dans l'expectative. Comment se positionne-t-on par rapport à cette attente, ce désir, cet espoir ou ce manque ? Ces questions-là conditionnent grandement l'écriture.

Personnellement, je perçois nettement cette possibilité d'entrer sur la scène du théâtre sur le mode d'une délégation d'examen. Il y a quelqu'un qui est délégué très provisoirement pour conduire une mise en examen d'un tout petit fragment de réel, et ce faisant inventer un peu de réalité. La proposition dramatique « entre » en scène 
en venant de la salle et pas d'un lieu mystérieux, inconnu, d'un arrière-monde qui serait la coulisse, la loge, etc. Elle est l'émanation de l'assemblée. Ça change beaucoup de choses. On part d'une forme de curiosité ou d'appétit de l'assemblée, dont fait partie l'écrivain au même titre que les acteurs et les spectateurs. Et ce geste extraordinairement impliquant de se lever du gradin, de prendre pied sur le plateau, et de se retourner vers les spectateurs pour faire une proposition, n'est pas sans conséquence sur la manière dont on commence.

C'est un peu comme dans un club de jazz, le musicien est au bar, il discute, salue les amis... Il est où tout le monde est. Et à un moment, il dit « tu m'excuses, il faut que j'y aille », et il va prendre pied dans l'espace de l'action. Ça m'a toujours bouleversé. Je sais que je me bouleverse d'un rien, mais c'est toujours ça de gagné sur le néant... C'est bouleversant, car c'est un semblable, un être humain tout à fait ordinaire qui est avec moi et qui, prenant place devant un piano, est capable de produire une rupture sensible considérable. Puis je le retrouverai pour boire une bière à l'issue du concert.

AdD : Je voudrais revenir sur ce très beau mouvement de l'acteur qui vient du public, et qui se retourne. Ce que je racontais sur Nathalie Dessay, Violetta, les coulisses, tout ce fantasme de ce qu'il y a derrière sur lequel Sivadier avait travaillé, ça me fait penser aussi au livre de J.-J. Schuhl que j'adore, qui s'appelle Ingrid Caven (2000). Ingrid Caven était l'égérie de Fassbinder et sa compagne. Dans ce livre, il essaye d'expliquer ce qu'est une présence sur scène. Il prend cet exemple : Ingrid Caven sort de sa loge et va jouer. Schuhl décrit tout ce chemin souvent assez tortueux entre les loges et la scène, souvent émaillé de choses invraisemblables. Elle emprunte à un moment donné une sorte de passerelle et bute avec son soulier dans une élingue de projecteur. Elle prend la chaînette et s'improvise un bracelet, avant d'entrer en scène avec sa robe Yves Saint-Laurent et son petit bracelet. C'est toute cette fantasmagorielà, l'entrée en scène.

Je repense aussi à une autre entrée de scène fulgurante, qui est complètement fantasmée, qui est celle du film de Dahan, La Môme (2007), la séquence dans laquelle Edith Piaf apprend la mort de Marcel Cerdan. Elle est affolée dans sa chambre d'hôtel. Il y a un plan séquence où elle rentre sur scène, dans la continuité de cette scène de chambre. C'est quelque chose d'assez extraordinaire. Cet enchaînement pose la question : d'où vient l'acteur? Est-ce qu'il vient des loges ou du monde? Ou bien estce que l'acteur est déjà là?

Dans $L a$ Traviata par exemple, les acteurs étaient déjà là, ce que Sivadier pratique beaucoup, mais l'héroïne n'était pas là. Elle faisait une vraie entrée, hyper classique. Et c'est cet équilibre qui était beau. Mais ce type d'entrée ne fonctionne qu'avec un mythe. C'est la star, qu'on attend, qui s'est maquillée, préparée, qui a pensé à des dizaines de détails. C'est ce qui est beau dans le livre de Schuhl, ce qu'il dit de la présence sur scène, qui n'est que détails : le bon choix pour l'œil, pour le costume, etc. Ça n'est pas quelque chose de divin. Mais mis bout à bout, ce sont des détails qui font une star ou une attitude de star.

EC : Certaines pièces de Thomas Bernhard débutent par une espèce de monologue ou de tirade, où l'on sent que depuis des années, des décennies, le personnage est pris dans un univers mental du ressassement. Quoi qu'on fasse, plus on avance dans ce ressassement, plus on a l'impression que le personnage a toujours été là et que lorsqu'on quittera le théâtre, il y sera encore, pour un siècle ou deux. C'est bien sûr 
très lié au fait que Bernhardt ne veut pas sortir de scène la question du passé nazi de l'Autriche. Donc on rentre en scène, et c'est toujours la même chose, toujours la même histoire, et vous allez en bouffer jusqu'à la fin du monde, je ne lâcherai jamais le morceau!

AdD : Et là il n'y a pas d'entrée en scène. Dans Des Arbres à abattre, que je viens de voir, l'acteur s'installe dans le noir. Donc ça ce n'est pas vraiment une entrée de scène.

Ph.D : Mais toutes les pièces de Bernhardt commencent comme ça !

AdD : Et on allume, il est dans son fauteuil...

EC : Et c'est reparti.

AdD : Ça commence mais il n'y a pas d'entrée de scène.

Ph.D : Ça existe de toute éternité.

$\mathrm{EC}$ : Ça commence moins que ça ne recommence. Il y a une inscription matérielle et concrète du point de vue qui s'énonce, simple, lisible, palpable. Une matérialité de la pensée.

Ph.D : Pour nous, comédiens, l'entrée s'envisage aussi comme une décision. On décide d'entrer, mais on peut aussi décider de ne pas entrer, décider que l'entrée vient d'avoir lieu, et qu'il suffit de se retourner, et de commencer. Cela constitue malgré tout une entrée aussi, un début en tous les cas, un commencement. Et peut-être que cela a beaucoup à voir avec la défiance de la fiction et du personnage. Qui rentre en scène ? Cela devient quand même très compliqué de croire qu'un personnage vient de je ne sais trop où. Cette convention-là devient difficile à vivre comme spectateur.

AP: II me semble que dans ce que vous dites, l'entrée en scène est vraiment l'affaire de l'acteur beaucoup plus que celle du metteur en scène?

Ph.D : C'est une question de théâtre. C'est quoi cet espace, comment il est occupé, qu'est-ce qu'il est, qu'est-ce qu'il incarne ? Comment on décore l'espace. Tout est lié. On peut faire des entrées si c'est des toiles peintes derrière. C'est un tout. Moi je peux parler des entrées pour le département, comment on travaille dans nos salles, comment on se sert des portes ou pas, comment on peut se convoquer et se dire que ça commence et qu'on le voit et qu'on le sent et qu'il n'y a pas besoin d'entrer. Donc oui, les acteurs, les auteurs, les scénographes, tous se posent la question de cet espace-là. C'est une question d'espace.

$\mathrm{EC}:$ On n'entre pas en scène une fois pour toutes. À chaque nouvelle action, nouvelle séquence, se repose la question de l'entrée en scène.

$\mathrm{AP}$ : Est-ce que c'est la première entrée en scène qui compte ou bien toutes les entrées sont importantes?

EC: Quelle raison on se donne de commencer une scène de telle façon plutôt que d'une autre ? C'est toujours un peu compliqué. Mais aussi : comment on sort de scène, de la scène en tant qu'unité dramaturgique, de la séquence. C'est très curieux car, dans la vie courante, quand quelqu'un sort d'une pièce, il peut lui arriver de dire au revoir, mais il est rare qu'il prenne congé sur une chute bien sentie, une ultime élégance. En même temps, quelle raison pourrait-on se donner de quitter la pièce au milieu d'une phrase? Qu'est-ce qu'on lègue aux acteurs lorsqu'on interrompt la scène au milieu d'une phrase et qu'on passe à la scène suivante? On lègue au plateau un silence problématique. On va devoir passer par un noir, traiter le problème... On a 
tendance à perdre de vue qu'on n'est pas au cinéma, et que les choses ne peuvent pas s'agencer avec un bout de scotch, ou en assemblant deux fichiers numériques. On a des corps dans un espace, dans une lumière, engagés dans des rapports concrets.

AP : Est-ce qu'on peut décider en tant qu'écrivain de ne pas se préoccuper de la question matérielle de l'entrée et de la sortie et de laisser cela au metteur en scène et à l'acteur? Je pense par exemple à des entrées problématiques comme celles des spectres ou des fantômes

EC : Sans doute, mais à condition que cette confiance et cette liberté ne masquent pas une forme de frivolité littéraire, ou une désinvolture consistant à créer des problèmes qui placeront l'écriture dans une prééminence ostentatoire. C'est bien gentil, cette tentation de démarrer toutes les scènes in medias res, mais il ne faut pas se plaindre ensuite que les spectacles sont lourds parce qu'ils sont hachés de noir (et lestés de musique pour éviter d'entendre les acteurs se replacer dans le noir.)

Ph.D : Je me demandais du coup s'il y avait des entrées impossibles aujourd'hui. Par exemple, une entrée de quelqu'un qui vient de la pluie, avec un imperméable, un parapluie et tout dégoulinant. Ça n'existe plus des entrées comme ça, ça ne s'écrit pas, ça ne se joue pas, ça ne se scénographie pas. Et pourtant le théâtre en regorge. On rentre avec un parapluie, on le secoue, l'air de dire : "Ah mon dieu, quel temps !", on a un peu de flotte sur la gueule, on essaye de faire croire à cette météo dehors. Mais ça va avec un certain décor et une certaine dramaturgie. Plus personne ne le ferait. On résoudrait cela différemment dans la mise en scène.

AdD : Ça me fait penser à Pommerat. Il n'y a quasiment pas d'entrées de scène chez Pommerat. Ce sont des apparitions, après avoir modifié le décor et installé les acteurs dans le noir. D'une manière un peu systématique d'ailleurs: on est censé ne rien entendre, ne rien voir, sauf que notre œil et notre oreille s'habituent. Au bout d'une heure, on distingue dans le noir la lueur des scotchs qui marquent les positions des acteurs et des accessoires. Voilà quelqu'un qui ne veut plus du tout parler d'entrée en scène. Ou alors est-ce qu'on peut encore parler d'entrée en scène? La lumière se rallume et c'est une entrée en scène?

Je voulais revenir également sur Oriza Hirata. Dans Nouvelles du plateau S, Hirata avait inventé une sorte d'espace-carrefour dans une clinique, où se croisaient les malades, le personnel soignant et les visiteurs extérieurs. Il travaillait beaucoup le tuilage des scènes: une scène se déroulait, une autre entrée survenait, commençait une autre scène pendant cette première scène. Il y avait un effet de réel très beau. Et cet effet tenait beaucoup aux entrées et aux sorties, parce que dans la vie les entrées et les sorties sont totalement aléatoires, surtout dans les espaces publics. Il y a un peu de Tchekhov dans ces spectacles, car chez Tchekhov il y a énormément d'entrées et de sorties. Sur Nouvelles, le travail scénographique était passionnant parce que c'était vraiment un enjeu. Dans d'autres spectacles, soit il n'y a aucune entrée et aucune sortie, les écrivains disent "noir ", et c'est un problème. Ça peut être super de faire cela, mais si c'est un procédé, c'est pénible, notamment pour les scénographes. Quand tu passes d'une pizzeria à une chambre à coucher, il est difficile de faire autrement qu'avec une tournette. 
Nouvelles du Plateau S, Oriza Hirata / Laurent Gutmann

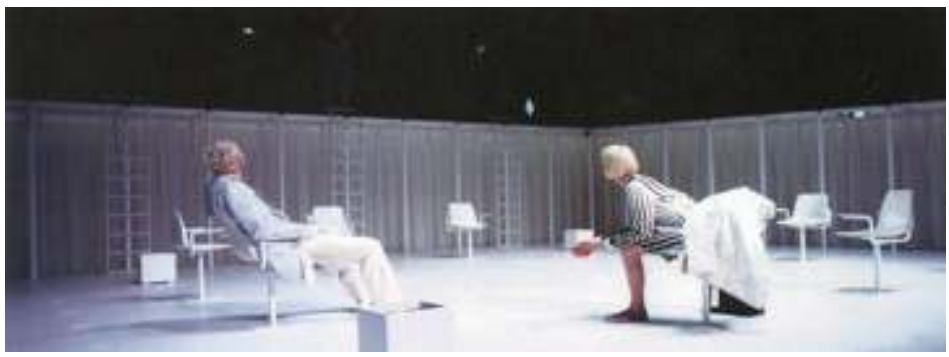

Scénographie Alexandre de Dardel (2003)

Alexandre de Dardel

EC : Nombre de jeunes écrivains dramaturges sont persuadés d'écrire pour le théâtre alors qu'ils écrivent manifestement pour le cinéma ou la télévision. C'est très compliqué de défendre cette matérialité du théâtre, sans avoir l'air de tirer les écrivains en arrière vers un académisme supposé ou un art pompier. C'est compliqué de repérer quels sont les éléments constitutifs de l'opération théâtrale, qui fait qu'en dépit de son archaïsme, ça reste un art pertinent pour le XXI ${ }^{e}$ siècle. Mais il faut se réapproprier cette singularité.

AP: Alexandre, est-ce que vos étudiants font parfois des décors impraticables, dans lesquels ils n'ont pas pensé la présence de l'acteur, ses entrées et ses sorties?

AdeD : Oui, cela arrive bien sûr. Lors d'un atelier avec Anne Alvaro par exemple, un étudiant très féru de vidéo a abouti à un projet dans lequel, même si c'était très impressionnant, Anne disait qu'elle ne pourrait rien faire là-dedans. Certains projets sont plus de l'ordre de l'installation que du décor de théâtre. C'est toute l'ambiguïté qu'il y a dans la scénographie, dans sa relation avec l'installation. Je suis frappé de voir que dans les arts plastiques d'aujourd'hui, le théâtre est très présent, mais c'est un théâtre de la désincarnation. Je pense par exemple à la dernière exposition de Sophie Calle à Avignon sur la mort de sa mère. C'est très théâtral. Sauf qu'il n'y a personne. D'autant plus que sa mère est morte. Donc cela raconte l'absence. Mais tous les codes de théâtre sont présents : l'espace, le son, la lumière. Sauf qu'il n'y a pas d'acteur. Mes étudiants sont très attirés par cette théâtralité sans personne, mais nous sommes à l'ENSATT, et nous devons travailler avec les acteurs et les écrivains.

EC: D'une façon concomitante, la situation qui est faite à l'écriture très contemporaine a fini, par la prolifération un certain nombre d'instances de mises en lecture, par faire revenir la mode de la pièce écrite pour être lue, la « lesen stücke ». Exit les problèmes de pizzeria et de chambre à coucher ! L'indication scénique suffit, l'imaginaire est parfaitement convoqué - provoqué. C'est évidemment le cas par exemple des Derniers jours de l'humanité de Karl Kraus, où les morts se relèvent d'entre les morts, où des centaines de chevaux sont faits prisonniers des glaces, etc. Dès l'écriture, il est clair que la pièce ne sera jamais jouée, mais lue et oralisée. La probabilité que la pièce voie le jour dans un espace scénique est très faible. D'où une attention flottante aux entrées et sorties, puisque dans une lecture, entrer/sortir, c'est tout bonnement parler / se taire.

AdD : De plus en plus on dit les didascalies. Donc qu'est-ce que ça veut dire pour le scénographe? Que c'est plus efficace s'il y a un acteur et pas de décor? 
Ph.D : C'est comme si l'entrée était le symptôme même de cette faillite-là. Comme si on renonçait au fait de croire qu'on entre supposément de quelque part. Mais d'autres éléments sont en faillite. Je n'emploie par exemple plus jamais le mot de personnage, je lui préfère celui de rôle. Une fois que l'acteur va s'approprier le rôle, cela pourra faire un personnage, mais cela ne nous regarde pas. Cela regarde le spectateur. Il n'y a pas de personnage vers lequel aller. L'acteur ne peut que s'approprier un rôle. Mais du coup, si le personnage n'existe pas, il ne peut pas venir d'ailleurs non plus, et donc il ne peut pas rentrer. C'est logique.

AdD : Sauf s'ils viennent vraiment d'ailleurs. Sauf si ce sont des fantômes, s'ils viennent d'un autre siècle. Là, je travaille sur Six personnages en quête d'auteur, et c'est difficile de faire entrer les personnages. On voulait les faire entrer par le décor dans lequel les personnages répètent. Et puis on s'est aperçu que ça ne marchait pas, donc on les fait entrer par la cage de scène, par le plateau.

EC : J'ai écrit et mis en scène une pièce intitulée La Révolte des Anges ${ }^{1}$, qui imagine la rencontre post-mortem de Jean-Michel Basquiat, Chet Baker et Bernard-Marie Koltès. Virée fraternelle dans les limbes... Dans la mise en scène que j'en ai proposée au Théâtre de la Colline, le dispositif était bi-frontal. Il y avait un plateau surélevé qui figurait l'espace de leur rencontre, donc l'espace du jeu, et un espace intermédiaire entre ce plateau et les gradins. Il fallait donc que les acteurs, pour entrer en scène, viennent d'une sorte de "là-bas» et passent par une espèce de non lieu. J'avais suggéré que les déplacements initiaux dans cette zone pourraient être considérés en quelque sorte comme extra-diégétiques. Et Carlo Brandt, qui jouait Chet, me disait: «non, moi je serai Chet dès que j'apparaîtrai ». Mais ce n'était que lorsqu'il prenait pied sur le praticable que ça faisait une différence. Il y avait un espace fortement ritualisé. Et c'est le fait de prendre pied dans cet espace qui constituait l'entrée - quoi qu'en eussent les acteurs. 


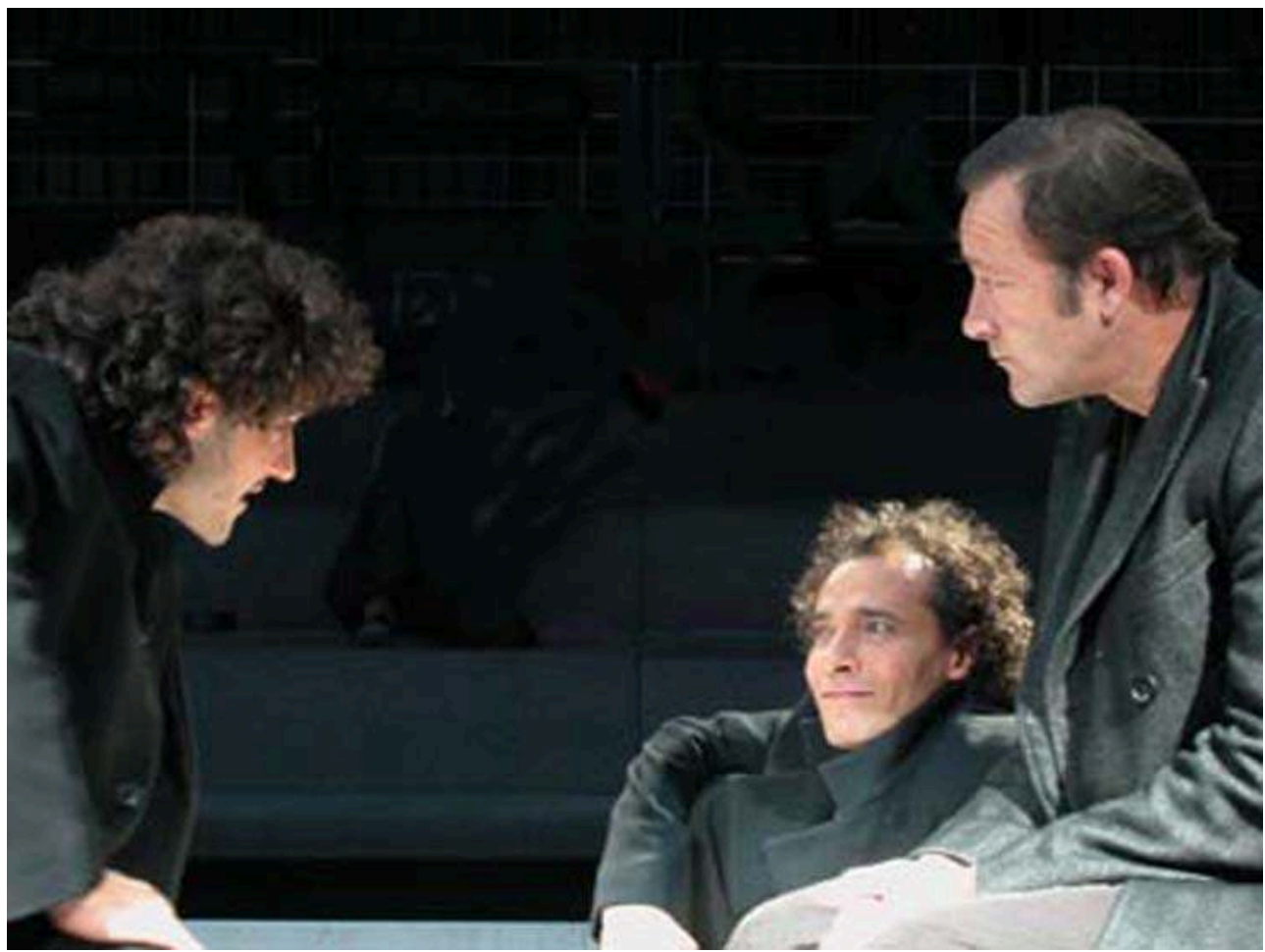

Thierry Blanc (Le Desperado Joyeux), Jean-Louis Loca (L'Enfant Radiant) et Carlo Brandt (Le Prince de la Félure), dans La Révolte des Anges, de et par Enzo Cormann.

Nicolas Boudier

AP: On parlait d'entrée en scène impossible, mais on pourrait peut-être parler de ces entrées où l'acteur incarne, endosse, exécute, comme vous voulez, un personnage ou un rôle extrêmement joué. Qu'est-ce que ça fait à l'acteur de rentrer dans le personnage d'Hamlet par exemple, plutôt que de créer un rôle contemporain ? Est-ce que ça change quelque chose sur le travail de l'entrée en scène? Est-ce qu'on se positionne par rapport à d'autres entrées en scène?

Ph.D : Je ne crois pas que cela modifie l'entrée en scène. Cela modifie tout le parcours d'un rôle qui a été joué et archi-joué. Ce sont les références.

EC : C'est la question du présent. Comment remettre le rôle au présent. En entrant en scène, l'acteur remet les pendules à l'heure, il positionne la barre du présent. À partir de là, nous ne sommes pas censés savoir ce qui va se passer. Si je passe mon temps à penser à ce pauvre con d'ÆEdipe qui ne se rend pas compte qu'il enquête sur sa propre perte... Donc je suis invité en tant que spectateur à rejoindre le présent du rôle - et donc l'aveuglement.

Ph.D : Je trouve beaucoup plus difficile de faire entrer Pierrot et Charlotte que de faire entrer le Commandeur, parce que faire entrer des paysans sur scène et savoir d'où ils viennent, ça c'est difficile. Mille fois plus problématique que de faire entrer le Commandeur ou le Spectre d'Hamlet. Parce qu'alors la crédibilité de la langue paysanne au théâtre, d'où ils viennent concrètement, comment ils sont habillés, c'est beaucoup plus compliqué que de croire à un mort. La question est toujours d'où ils viennent. Si c'était encore un pur hors-scène, des coulisses donc, ça irait. Mais le problème c'est bien de faire croire à un moment donné que ce n'est pas la coulisse, que Pierrot vient de chez lui et qu'il va y retourner, et que Charlotte n'habite pas loin. 
$\mathrm{AP}$ : Est-ce que l'entrée en scène a tendance à disparaître des scènes contemporaines ?

Ph.D : Je ne sais pas du tout vers quoi le théâtre va aller, si c'est cyclique. Il y a des mutations profondes dans le théâtre. J'ai l'impression qu'il ne reste que le carré de plateau comme espace de la fiction possible, et que la coulisse a disparu, le rôle, le costume, qu'on va de plus en plus vers une narration, un théâtre épique. J'ai du mal à imaginer le retour de la fiction, de la coulisse, des entrées de personnages.

\section{NOTES}

1. Le texte date de 1998 et la mise en scène de 2006. Création au Théâtre de la Colline.

\section{RÉSUMÉS}

Afin d'interroger l'entrée en scène à la fois selon différents domaines de l'art du théâtre (écriture, scénographie et jeu), et en relation avec la question de la formation dans ces disciplines, cet entretien croise les voix de trois artistes et directeurs de département de l'ENSATT (Ecole Nationale Supérieure des Arts et Techniques du Théâtre). Il est complété par l'entretien avec Daniel Deshays sur la question de l'entrée en scène du sonore.

\section{INDEX}

Mots-clés : formation, école, dramaturgie, écriture, jeu, scénographie 